Crise(s) dans le monde ibérique et ibéro-américain

\title{
La Antoniana Margarita (1554) de Gómez Pereira et la crise de l'aristotélisme scolastique
}

\section{Christian Andrès}

\section{(2) OpenEdition}

1 Journals

\section{Édition électronique}

URL : https://journals.openedition.org/cher/3970

DOI : $10.4000 /$ cher.3970

ISSN : 2803-5992

\section{Éditeur}

Presses universitaires de Strasbourg

\section{Édition imprimée}

Date de publication : 1 décembre 2015

Pagination : 249-260

ISBN : 978-2-86820-913-9

ISSN : 1968-035X

\section{Référence électronique}

Christian Andrès, "La Antoniana Margarita (1554) de Gómez Pereira et la crise de l'aristotélisme scolastique », reCHERches [En ligne], 15 | 2015, mis en ligne le 01 décembre 2021, consulté le 16 décembre 2021. URL : http://journals.openedition.org/cher/3970 ; DOI : https://doi.org/10.4000/cher 3970

\section{cc) (i) (2)}

Ce(tte) œuvre est mise à disposition selon les termes de la Licence Creative Commons Attribution -

Pas d'Utilisation Commerciale - Partage dans les Mêmes Conditions 4.0 International. 


\title{
La Antoniana Margarita (1554) de Gómez Pereira et la crise de l'aristotélisme scolastique
}

\author{
Christian Andrès \\ Université de Picardie
}

Nihil praeter veritatem impulisse me, ut praesens opus conficerem ${ }^{1}$. Gómez Pereira (1500-1558?)

Le $\mathrm{XVI}^{\mathrm{e}}$ siècle espagnol - à y regarder de près - n'est pas aussi monolithique qu'on a bien voulu le croire, et ce n'est pas José Antonio Maravall qui nous démentirait. Alain Guy non plus, qui en 1983 pouvait écrire ceci:

Contre la scolastique, majoritaire encore, plusieurs écrivains vont se dresser, qui diffusent, dans l'ombre ou publiquement, leurs messages audacieux [...]. Parmi les autres réfractaires, les philosophes biologistes jouèrent un rôle non négligeable; grâce à ces médecins dont la curiosité et l'activité étaient universelles, la modernité va progresser, insensiblement mais sûrement, en dépit de la répression du SaintOffice (Guy 1983: 102-103).

«Philosophe biologiste», "physiologiste éclairé» ou, tout simplement, "médecin philosophe», Gómez Pereira - dont beaucoup de traits biographiques restent encore énigmatiques - a pu être qualifié magnifiquement par l'érudit

1 Toutes les citations de la Antoniana Margarita seront faites à partir de l'excellente et assez récente édition bilingue (latine-espagnole) de José Luis Barreiro Barreiro, publiée par la Fundación Gustavo Bueno, Universidade de Santiago de Compostela, 2000, qui est aussi la reproduction fac-similé d'un exemplaire de la $2^{\mathrm{e}}$ édition l'édition madrilène de 1749 - rarissime exemplaire conservé à la bibliothèque de l'Université de Saint-Jacques de Compostelle. La première édition est datée de 1554 et fut imprimée à Medina del Campo. Ici, il s'agit d'un court extrait de l'" Avertissement au Lecteur" qu'est son Ad lectorem scopus authoris in conficiendo opere, compris entre la page XXXVI et XXXVII. Barreiro Barreiro traduit ainsi: «nada, excepto la verdad, me ha impulsado a escribir la presente obra» (XXXVII).

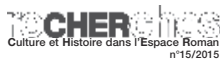


Menéndez Pelayo de "pensador independiente y ciudadano libre de la república de las letras» (Menéndez Pelayo 1964: 245).

Mais, au-delà des appréciations plus ou moins enthousiastes ou sévères qui ont pu être formulées sur son œuvre, au-delà d'une sorte d'occultation dont il a pu pâtir dans l'histoire des idées, quel rôle jouerait ce médecin-philosophe dans l'histoire de la philosophie et dans ce qui peut être appelé «la crise de l'aristotélisme scolastique» ? Précisons tout de suite que, dans ce domaine, parler de «crise» veut d’abord dire que la scolastique médiévale a connu diverses phases sur une longue durée, avec un apogée à la fin du XII siècle-fin XIII ${ }^{e}$ siècle - «la grande scolastique»-, suivie d'un repli sur elle-même, une moindre vigueur aux $\mathrm{XIV}^{\mathrm{e}}-\mathrm{XV}^{\mathrm{e}}$ siècles et, pour finir, une entrée en décadence sous les puissants coups de boutoir de l'humanisme et de la Réforme dès le début du Xvie siècle, auxquels s'ajoutent pour l'Espagne les brèches ouvertes par "les philosophes critiques", c'est-à-dire des penseurs indépendants comme Juan Luis Vives, Gómez Pereira, Miguel Sabuco, Juan Huarte, Francisco Sánchez - «el Escéptico»-. Rappelons, par exemple, ce qu'Érasme a pu écrire: «Quelles relations peut-il y avoir entre Christ et Aristote? $\gg^{2}$, tandis que Luther, pour sa part, n'a que faire d'Aristote: «En bref, tout Aristote est à la théologie ce que les ténèbres sont à la lumière. ${ }^{3} \mathrm{Et}$ ce préambule n'avait pour toute ambition que de suggérer d'emblée la complexité que présente la scolastique entendue comme système de pensée et méthode d'enseignement, fonctionnant sur plusieurs siècles, ainsi que la grande difficulté à analyser la "crise» dans ces conditions, et cela même en se limitant au seul $\mathrm{XVI}^{\mathrm{e}}$ siècle et, surtout, en ne nous intéressant qu'à un seul de ses penseurs.

La Antoniana fut publiée à Medina del Campo, en 1554 et son titre - ou du moins ses deux premiers mots - est déjà à lui seul une curiosité novatrice pour une œuvre philosophique. En effet, cette œuvre tout à fait exceptionnelle s'intitule exactement: Antoniana Margarita, opus nempe physicis, medicis, ac theologis, non minus utile, quam necessarium, per Gometium Pereyram, medicum Methymne Duelli, quee Hispanorum lingua Medina del Campo appellaturt. Titre insolite parce que l'auteur l’a composé à partir du prénom de son père et de sa mère, et qu'il éprouve le besoin de se justifier dans sa «Razón del título de esta obra» (Gómez Pereira 1554: XXXV). C’est, nous dit-il, qu'il a longtemps hésité et n’a pas trouvé finalement le titre adéquat. En fait, il aurait bien appelé son volumineux traité «Paradojas», mais son livre navait pas pour objet le paradoxe. Il n'a d'ailleurs pas trouvé d'autre titre plus correct ni plus convenable, semble-t-il, et pour ne pas être ambigu - Gómez Pereira semble détester toute ambiguité dans la formulation écrite et le raisonnement analytique - il a donc opté pour le nom

2 Dans sa lettre à Martin Drop, 1515.

3 Thèses contra scholasticam theologiam, 1517.

4 Traduit ainsi par Barreiro Barreiro: «Antoniana Margarita, obra, no menos útil que necesaria, que trata sobre temas físicos, médicos y teológicos, por Gómez Pereyra, médico de Methymnae Duelli - que en la lengua española se denomina Medina del Campo». 
de ses parents, Antonio et Margarita. Ce qui peut certes être pris pour un acte de piété filiale, mais qui, en réalité, traduit surtout sa propre incapacité à trouver le bon titre, celui qui refléterait bien toute son œuvre... Il est vrai que, dans le cas de la Antoniana, réduire le titre à quelques mots est une gageure, puisque ce livre magistral et novateur n'est un traité méthodique ni de psychologie, ni de physique, ni de métaphysique, s'il est un peu tout cela à la fois, mais essentiellement, selon les propres termes de Menéndez Pelayo, «un libro de controversia, una serie de paradojas» (Menéndez Pelayo 1887: 189). Et ce qui est remarquable, parmi d'autres aspects, c'est que notre auteur va utiliser les "propres armes» de la Scolastique pour les retourner contre elle, puisqu'il a recours avec talent et finesse à la méthode scolastique, soit, comme le dit encore Menéndez Pelayo, «el arte de separar, distinguir y subdividir hasta lo infinito [...]» (Menéndez Pelayo 1887: 173), pour réfuter ou disqualifier principalement maintes opinions d'Aristote et de ses commentateurs. Voilà déjà une modalité de crise, lorsqu'un philosophe emploie une méthode argumentative et herméneutique dominante - soit ici l'analyse scolastico-thomiste - pour dénoncer les assertions qui lui ont paru fausses, sans trop respecter les auctoritates, mais en ayant recours, systématiquement, au critère de l'expérience et de la raison.

D'Aristote, il est très souvent question dans la Antoniana, et de toute évidence Gómez Pereira - qui étudia la philosophie et la médecine à l'Université de Salamanque $^{5}$ - en fut un grand lecteur et un fin critique, voire contradicteur, comme notre examen des références aristotéliciennes contenues dans la Première Partie $^{6}$ de cet ouvrage peu commun, suffirait déjà, à lui seul, à le prouver: sur 178 pages environ, nous voyons citer pas moins de 130 fois le nom d'Aristote et/ou celui d'un de ses traités, avec le plus souvent le commentaire d'une thèse ou d'une assertion du Stagirite; Gómez Pereira la discute, citation à l'appui, et l'examine quand l'autorité de ce grand philosophe ne se voit pas mise en doute - et parfois même avec quelque suffisance - lorsqu'une contradiction ou une ambiguïté sont impitoyablement décelées. Quant aux œuvres d'Aristote ici évoquées, nous n’en avons pas trouvé moins de vingt, nombre en soi assez impressionnant ${ }^{7}$. Nous

5 Ce nominaliste fut disciple de Juan Martínez Guijarro, plus connu sous le nom redouté de Silíceo.

6 Nous parlons ici de "première partie» de la Antoniana en partageant le critère du récent éditeur José Luis Barreiro Barreiro qui s'en explique dans son «Estudio preliminar», $\$ 47$. Celui-ci précise que l'ordre des thèmes abordés et traités (si des retours et des interpolations ne sont pas évités tout au long du discours) est le suivant: la thèse de l'insensibilité animale; l'explication du mouvement animal; les modes de la pensée; les questions ontologiques, psychologiques, anthropologiques et cosmologiques.

7 Nous pouvons en dresser la liste suivante: De Anima, 3; De Historia Animalium; Analíticos Posteriores; Perihermenias; De Physica; Retóricos; Éticas; De Somno et Vigilia;De Partibus Animalium; Metaphysica; De Phantasmatibus; Predicamentos;De memoria et Reminiscentia; Tópicos; De Generatione et Corruptione; De Meteorologia; De Mundo ad Alexandrum; De Caelo et Mundo; Sensu et Sensato; De Generatione Animalium. 
pouvons encore préciser que sur ces vingt écrits d'Aristote, les trois ouvrages suivants sont les plus cités et analysés, le premier l'emportant nettement sur les deux autres: De Anima (37 fois); De Physica (17 fois); De Metaphysica (14 fois). Ajoutons enfin la considération de notre Philosophe de Medina del Campo pour l'érudition aristotélicienne: le livre III du traité De Anima d'Aristote sera en outre l'objet d'une savante et féconde paraphrase - «que difiere de la exposición de todos los demás autores»- pour commencer la Seconde Partie de la Antoniana ${ }^{8}$.

Revenons à la méthode scolastique ${ }^{9}$ elle-même, et aux formes qui lui sont propres (commentaires, questions disputées, questions quodlibétales, sommes): l'intention qui l'anime est nettement didactique.

Gómez Pereira - fin dialecticien lui-même, et logicien remarquable - va donc recourir à la méthode scolastique qu'il connaît bien, mais à sa manière, en procédant de façon très personnelle et atypique. C'est-à-dire qu'il peut soit réfuter d'entrée de jeu une thèse communément admise par «L'École», comme il dit, et argumenter ensuite, soit paraître respecter la méthode usuelle tout en la «parasitant» subtilement de l'intérieur, en pratiquant souvent d'interminables digressions, et tout cela afin de pouvoir avancer et défendre ses propres thèses paradoxales, selon le sens suggéré par l'étymologie grecque du terme ${ }^{10}$. La volumineuse Antoniana Margarita ne manque certes pas de "paradoxes" et, en fait, les thèses paradoxales qui y sont contenues, explicitées et défendues, se présenteraient selon nous comme une tentative audacieuse et cauteleuse à la fois pour élaborer une nouvelle et originale doctrine philosophico-psychologique humaine et animale en rupture avec bien des connaissances admises jusqu'ici. Pour ne prendre qu'un exemple, il suffira de constater comment Gómez Pereira procède dès le début de son ouvrage lorsqu'il s'attaque à l'opinion orthodoxe selon laquelle les animaux - les "brutes» - ont une sensibilité tout comme l'homme, considérations et arguments qui préludent de toute évidence avec quelque quatre-vingts ans d'avance à la fameuse thèse cartésienne des «animauxmachines », c'est-à-dire de l'automatisme des bêtes. En effet, pour Gómez Pereira qui est le premier auteur dans l'histoire de la pensée à nier la sensibilité animale, le paradoxe, voire l'erreur, c'est plutôt le préjugé si généralement répandu qui attribue la sensibilité aux animaux, et qui prendrait sa source dans un passage mal interprété du troisième livre De l'Âme, d'Aristote. Mais voyons auparavant comment notre médecin-philosophe s'y prend: avant d’en venir à la question qu'il lui faudra examiner - la sensibilité ou non des animaux - Gómez Pereira se présente tout d'abord comme un témoin direct de ce qu'il va narrer, et comme

8 La «Segunda Parte» comprend, outre cette Paraphrase, le fameux Tratado sobre la inmortalidad del alma plusieurs fois annoncé dans la «Primera Parte»; les Objeciones de Miguel de Palacios a la Antoniana Margarita; la Apología de Gómez Pereyra a ciertas objeciones de las múltiples paradojas de la Antoniana Margarita.

9 «Scolastique» vient de scola, «école».

10 Paradoxe vient du grec para, "contre», et doxa, «opinion», soit une opinion, une assertion ou une proposition qui va «contre l'opinion commune » et la vraisemblance. 
un écrivain à la recherche de la vérité objective. Dans son court préambule, il a la sage précaution de s'en remettre humblement à l'autorité du Souverain Pontife et de l'Église romaine au cas où il aurait commis quelque erreur involontaire. Cela dit, il expose tout de suite l'opinion commune en ces termes:

Se ha asumido por doctos y no doctos que la facultad sensitiva es común a brutos y a hombres - hasta tal punto que nadie duda sobre ello, como tampoco se discute el principio de «que cada todo es mayor que cada una de sus partes» (Gómez Pereira 1554: 1, col. 1).

L'auteur se dit alors opposé à la majorité des physiciens et affirme, avec superbe, ne pas chercher à les convaincre tant ils sont imbus de leur savoir et incapables de s'ouvrir à toute autre opinion que la leur. Et d'exprimer alors, contrastant fortement avec ce qui vient d’être dit, un sentiment orgueilleux d'égalité au moins avec les siècles passés, l'Antiquité, une fierté tout à fait caractéristique de la Renaissance:

Pero tengo la suerte de escribir en una época en la que casi todos los prepósitos de España son muy expertos en lo físico y en lo teológico, e incluso en derecho pontificio, hasta el extremo que no tienen nada que envidiar a los más doctos de otros siglos (Gómez Pereira 1554: 1, col. 2).

Et d'ajouter encore ceci:

Por todo ello, yo los considero como mis censores y me atreveré a disentir de lo que nadie antes fue capaz -ni de palabras, ni por escrito- sobre el axioma antes citado y que he propuesto con anterioridad, explicado en forma de paráfrasis (Gómez Pereira 1554: 1, col. 2-2, col. 1).

Gómez Pereira a donc clairement conscience d'être le premier penseur à refuser toute sensibilité aux animaux ${ }^{11}$, n'admettant pas «lâme sensitive» qu'Aristote accordait sans sourciller aux êtres vivants «irrationnels». Notons également, au passage, l’emploi du terme "axiome», qui veut dire en philosophie «vérité indémontrable», "principe premier tenu pour vrai»12. Autrement dit, qualifier d'«axiome» l'assertion admise par tous qu'il existe une sensibilité animale autorise Gómez Pereira à n’en pas donner la raison, et l'incite à paraphraser ce que veut dire une sensibilité commune aux «brutes» et aux hommes. Malicieusement, à notre avis, il commence par faire remarquer quadmettre la sensibilité chez les animaux équivaudrait à dire que les animaux voient ce que nous voyons en ayant recours comme nous à «la pure connaissance». Il fait alors ce raisonnement par l'absurde:

[...] si hubiera sido posible que los brutos hablaran, éstos habrían denominado al color que ven blanco como «album»-si hablaran en latín- o «blanco»-si

11 Pierre Bayle le confirme dans son Dictionnaire historique et critique, 1695-1697.

12 Le problème de l'axiome selon la logique, c'est qu'il est indémontrable, indéfinissable. Selon Edmond Goblot, «[...] tout jugement exprimant une nécessité, par exemple un axiome, est dans sa forme un jugement hypothétique. [...] Les axiomes sont des jugements dont il est impossible de donner la raison parce qu'ils sont premiers, et qui ne sauraient être évidents sans démonstration parce qu'ils sont synthétiques» (Goblot $1922: 324, \S 210)$. 
hablaran en español. Incluso si pudieran tocar una figura cuadrada, la habrían denominado con el concepto latino «quadratum» o con la expresión española «figura cuadrada». Y de la misma manera se servirían de los demás sentidos externos. (Gómez Pereira 1554: 2, col.1)

Pour Gómez Pereira, de toute évidence, les «brutes» ne sont pas semblables à nous dans la connaissance externe. Il montre aussi qu'il connaît l'opinion de certains qui refusent aux animaux l'existence de la «réflexion» sur les actes produits par les sens externes. C’est alors que, contrairement au schéma traditionnel de la quaestio en scolastique, l'auteur de la Antoniana escamote délibérément le recours obligé aux auctoritates et aux penseurs divers qui ont des opinions différentes de la sienne, et s'en explique ainsi:

[...] advierto a todos vosotros que no voy a rechazar la opinión de ningún autor, por grave que sea, con tal que no se trate de religión, y que me voy a apoyar únicamente en razones (Gómez Pereira 1554: 2, col. 1-2).

Il ne tardera pas alors à s'en prendre à l'autorité d'Aristote qu'il trouve bien peu fiable dans cette matière:

Porque, según pienso, éste escribe a propósito de una forma tan oscura que el mismo contexto puede ser considerado en un sentido o en el otro (Gómez Pereira $1554: 2$, col. 2),

défaut majeur aux yeux rationalistes - pour ainsi dire - de notre médecinphilosophe. Puis suivent quelques considérations iconoclastes comme celle-ci:

Pero, además olvidan que debe ser rechazada cualquier autoridad en asuntos que interesan a la especulación y no a la fe (Gómez Pereira 1554: 2, col. 2).

Et d'insister sur la légitimité qu'il y a à raisonner sans aucune contrainte «en el campo de la especulación y de la naturaleza» (Gómez Pereira 1554: 2, col. 2), autrement dit en dehors du domaine de la foi, puisque de cette manière les sciences peuvent progresser dans leurs découvertes. Enfin, Gómez Pereira résume pour son lecteur tout ce qui va suivre en ces termes:

Pero, para no hacer esperar a los que desean leerme, voy a explicar, lo más brevemente que me sea posible, en qué se diferencia lo característico del hombre del resto de los brutos. En segundo lugar, aportaré las razones en las que conste manifiestamente que los brutos son semejantes a nosotros en el sentir y que, de acuerdo con esto, se debe inferir necesariamente que no hay nada propio para nosotros -los hombres- que no sea común para ellos; ya que si de las operaciones de los brutos se extrae una, también necesariamente se deducen las restantes. En tercer lugar, expondré cuál es la causa del movimiento de los brutos y como se mueven. (Gómez Pereira 1554: 2, col. 2-3, col. 1)

Pour le dire succinctement, et en anticipant sur la démonstration qui suit et la réfutation de certaines opinions, il y a un enjeu de taille dans cette question de la psychologie animale, et dans cette psychologie comparée de l'animal et de l'homme, et c'est le suivant: si les animaux avaient une sensibilité commune à celle des hommes, s'ils nous ressemblaient également dans le raisonnement et l'intellection des universaux, où serait alors la différence entre eux et nous? Et qu'en serait-il de l'âme et de l'immortalité de l'âme? Lâme des animaux serait- 
elle alors immortelle comme celle de l'homme? Or, l'immortalité de l'âme doit rester le propre de l'homme - opinion qui est du domaine de la foi et ne peut être contredite - et elle doit donc être refusée aux animaux. Par ailleurs, dans cette question de la sensibilité animale, il faut savoir qu'Aristote concédait aux «brutes» lâme sensitive s'il leur déniait l'âme rationnelle. La thèse de Gómez Pereira - «Bruta carere sensu probatur $»^{13}$ - est donc opposée à celle d'Aristote et à celle de la philosophie scolastique en vigueur. Cependant, à propos de la cause qui expliquerait le mouvement des animaux, notre auteur admet l'intervention d'un instinct (ou quelque chose d'équivalent), et l'existence chez l'animal d'une certaine capacité d'apprentissage. Les bêtes auraient une âme divisible et périssable, soit une âme quantitative et mortelle. Rappelons maintenant que la thèse officielle partagée par presque tout le monde était celle de Luis Vives (De anima et vita, lib. II) pour qui les brutes avaient la sensation, l'imagination et la faculté estimative, soit l'instinct naturel, mais n'avaient ni la raison (âme intellective) ni l'immortalité. Il est vrai que la question de l'instinct est capitale en psychologie animale. Et qu'il faut bien distinguer entre la nature de l'instinct animal et l'intelligence humaine: si l'on reconnaît la conscience de la sensation, l'intelligence aux bêtes, alors c'est admettre que les animaux ont une âme semblable à celle de l'homme, c'est donc affirmer qu'ils sont de la même espèce que nous - «eiusdem esse speciei»-, et ce serait aboutir à une conclusion aussi absurde qu'impie. Mais les bêtes fuient le feu et l'ennemi: c'est qu'elles en ont une forme de connaissance, une relative faculté de raisonner - "discurrendi vim». Chez Gómez Pereira, il y a bien une tendance au mécanicisme et, tout compte fait, tout peut se réduire à un problème de mécanique, si une "propriété occulte» semble commune aux phénomènes dans les trois règnes de la nature. En bref, les animaux reçoivent des impressions, et c'est tout. Ils nont pas de sensibilité, ni de perceptions. Car seuls les hommes ont la sensibilité, les animaux n’ont que le mouvement. Menéndez Pelayo insiste sur l'originalité du système inventé par Gómez Pereira pour expliquer les opérations des animaux, système qui est étrangement semblable à celui de Descartes tel qu'il l'exposera dans son fameux Discours de la méthode, quelque quatre-vingt-trois ans plus tard... Et l'on peut même estimer que la thèse cartésienne des animaux-machines est plus simpliste que celle de Gómez Pereira - qui n’a jamais employé, lui, le mot d' "automate» dans son Antoniana - parce que Descartes, dans le fond, réduisait les animaux à de la matière assujettie aux lois du mécanisme universel.

Lorsqu'un philosophe est capable de penser par lui-même, de douter de ce qu'il croit savoir ainsi que des opinions d'autrui, fussent-elles des auctoritates prestigieuses, comme létait Aristote, lorsqu'un philosophe prenant grand soin de ne pas contredire ce qui touche au domaine de la théologie et de la foi catholique ne donne aucune limite à son activité spéculative si ce n’est le critère de la vérité, de l'expérimentation et de la conformité à la raison, il n'est pas étonnant que ses réflexions aboutissent à des conceptions originales et à des théories nouvelles,

13 Écrit en marge de la col. 2, et en italique dans l'édition fac-similé de 1749, p. 3. 
certaines d'entre elles pouvant même être très proches du matérialisme, comme le pensaient son contemporain Miguel de Palacios et plus récemment Menéndez Pelayo. En effet, le Philosophe de Medina del Campo propose également dans la Antoniana une théorie de la connaissance qui soppose à la théorie scolastique en vigueur, en estimant que la connaissance est directe, telle que l'expérience nous la montre, qu'elle est non distincte du sujet connaissant, et qu'elle n'a pas besoin des idées - «especies inteligibles» - ni d’images intermédiaires. Mais Gómez Pereira défendra bien d'autres thèses importantes: la connaissance commence par la sensation ${ }^{14}$; la sensation est différente de l'impression ou affection créée dans un organe (œil, oreille, nez...); elle ne provient pas d'un objet ni de la faculté sensitive, mais elle est une modification de lâme, un modus habendi de lâme. L'intellection - acte de comprendre - et l'intelligence sont une même chose, de même que l'intelligence n'est autre chose que l'essence de l'âme "actus intellectus idem cum anima». Le sens commun est une pure invention, il n'existe pas; l'ancienne classification des facultés de l'âme, celle de l'École, est artificielle et en partie fausse puisque l'imagination - «fantasía» - est une faculté intérieure non organique, donc non localisée, alors que la mémoire est une faculté organique et réside dans la partie postérieure de la tête (locciput). Il n'existe pas de distinction réelle entre la faculté sensitive et la faculté intellective: les universaux nous sont connus par abstraction, et ils nont de réalité que dans l'esprit; l'essence de l'âme, c'est la pensée (ou l'entendement), ce en quoi Gómez Pereira précède Descartes; de la notion des objets sensibles nous pouvons nous élever à celle des indivisibles (Dieu, les anges, les intelligences et les âmes). Enfin, pour nous limiter dans l'examen des nombreuses contributions théoriques, philosophiques et physiologistes de Gómez Pereira - et sans pouvoir nous pencher sur l'étonnante «démonstration» rationnelle de l'immortalité de l'âme dans le petit traité du même nom inclus dans la Antoniana - nous voulons évoquer la passionnante question du «cogito» déjà exposée dans cette œuvre, bien avant Descartes par conséquent. Certes, dans le cas français, le célèbre «je pense, donc je suis» (Discours de la Méthode, 1637) s'insère dans toute une réflexion qui a pour but d'asseoir des principes universels et certains pour parvenir à la vérité et donc philosopher avec justesse, en pratiquant le doute méthodique et en élaborant une méthode de pensée en quatre règles. Considéré par Hegel comme «le fondateur de la philosophie moderne», son fameux "cogito» s'est imposé et a presque totalement éclipsé le "cogito» pereirien qui, à notre avis, ne méritait pas pour autant de tomber dans les oubliettes de l'histoire de la pensée... Si l'on oublie un instant la portée énorme du «je pense, donc je suis» de Descartes, pour nous intéresser à son aspect formel, selon la logique classique et la syllogistique aristotélicienne, nous avons là un syllogisme imparfait, abrégé, parce que tronqué: il y manque, en effet, la majeure. C’est donc ce que lon appelle un enthymème, soit un syllogisme incomplet, dont

14 En ce sens, Gómez Pereira ne peut qu'être d'accord avec cette célèbre formule d'Aristote: «Tout ce qui est dans l'entendement a passé par les sens». 
on a supprimé une des deux prémisses. Autrement dit, Descartes n'exprime par là qu'une seule proposition antécédente (une mineure), et une proposition conséquente (la conclusion). Mais le plus grave, selon nous, c'est qu'un doute subsiste pour restituer la majeure absente du fameux enthymème cartésien. Fautil imaginer une proposition du genre: "pour penser, il faut être», ou: "Tout ce qui pense est (ou «existe», car Descartes lui-même semble hésiter entre ces deux termes), ou bien encore: "Si tout être qui pense est»? Dans le premier cas, nous aurions affaire à un syllogisme catégorique tronqué et dans le second cas, à un syllogisme hypothétique tronqué (ou encore un syllogisme conditionnel). Quelle que soit la majeure inexistante dans le fameux «je pense, donc je suis», tout repose sur une tautologie, une évidence, un axiome pour ainsi dire...

Voyons maintenant ce qu'il en est du "cogito" pereirien, à presque un siècle de distance. Il s'agit d'un passage du petit traité De l'immortalité de l'âme de la Antoniana Margarita, où le philosophe veut démontrer l'immortalité de lâme par l'existence d'une âme intellective qui non seulement connaît les choses extrinsèques, mais encore se connaît elle-même de manière intuitive. Son syllogisme latin est parfait: "nosco me aliquid noscere, \& quicquid noscit est, ergo ego sum» (Gómez Pereira 1554: 277, col.2) ${ }^{15}$. Ce qui, traduit par nous, veut dire: «je connais que je connais quelque chose, et tout ce qui connaît est, donc je suis». De fait, un tel syllogisme nous semble correspondre - sauf erreur - à un syllogisme de la troisième figure ${ }^{16}$, et du troisième mode (I A I), soit au Disamis des scolastiques.

S'il est possible de conclure dans une telle matière, nous dirons que la crise de l'aristotélisme scolastique est lisible dans la Antoniana Margarita de Gómez Pereira. Nous n'avons pu constater certaines modalités de cette crise quà travers l'examen et la remise en cause de diverses assertions du Stagirite ou de commentateurs comme Avicenne, mais d'autres critiques ou réfutations sont également formulées, notamment en ce qui concerne l'immortalité de l'âme (il trouve bien insuffisantes toutes les théories formulées avant lui, y compris celle de Platon dans le Phédon, et de saint Augustin dans les livres De Quantitate Animae et De Inmortalitate). Gómez Pereira est un redoutable dialecticien qui emploie les procédés mêmes de la scolastique contre les théories scolastiques, et fonde les siennes systématiquement sur l'observation. Il oppose résolument son expérience personnelle à celle des maîtres, à leur autorité, et entreprend de démontrer à l'occasion que leurs arguments sont fragiles, ou ambigus ou erronés. Le médecinphilosophe de Medina del Campo, empiriste invétéré, est encore l'auteur d'un syllogisme en latin qui précéda le fameux enthymème cartésien - cogito ergo

15 Il est dommage que le lecteur qui ne connaisse pas le latin ait droit en cette circonstance à une version en castillan tronquée, où il manque la mineure, puisque Barreiro Barreiro propose: "yo sé que conozco algo, luego existo ».

16 Soit un syllogisme où le moyen terme est le même (ou presque, dans notre cas: «nosco» et «noscit») dans deux propositions, et où la mineure doit être affirmative (A ou I), tandis que la conclusion est particulière (I ou O). 
sum - mais dont il ne fera pas le premier principe d'une nouvelle philosophie. Enfin, nous ferons nôtres ces propos admiratifs de Juan María Guardia sur la singulière avance que Gómez Pereira pouvait avoir sur son temps:

Il a précédé Bacon, Descartes, Spinoza, Locke, Leibniz, qui, ou se sont rencontrés avec lui, ou lui ont emprunté en grands seigneurs, peu reconnaissants envers leur créancier (Guardia 1889: 634).

\section{Bibliographie}

Goblot, E., 1922, Traité de Logique, 3e éd., Paris, Librairie Armand Colin.

Gómez Pereira, 1554, La Antoniana Margarita, Medina del Campo, ed. bilingüe de Barreiro Barreiro, J. L., 2000, Universidade de Santiago de Compostela, Fundación Gustavo Bueno.

Guy, A., 1983, Histoire de la philosophie espagnole, Toulouse, Publications de l'Université de Toulouse-le-Mirail.

Guardia, J. M., 1889, "Philosophes espagnols. Gómez Pereira», Revue philosophique de la France et de l'étranger, t. XXVIII, p. 270-291; p. 382-407; p. 607-634.

Menéndez Pelayo, M., 1964, La Filosofía española, Selección e introducción de Constantino Lascaris Commeno, Segunda ed., Madrid, Ediciones Rialp. Menéndez Pelayo, M., 1887, La ciencia española, T. II, 3e ed., Madrid. 